\title{
TAILOR-MADE COPOLYMERS FOR RESPONSIVE DRUG DELIVERY NANOSYSTEMS
}

\author{
$\underline{\text { Sébastien Cajot }}^{1}$, Christine Jérôme $^{1}$ \\ ${ }^{1}$ Center for Education and Research on Macromolecules (CERM), University of Liege, B6 Sart \\ tilman,B-4000Liege,Belgium.e-mail:s.cajot@ulg.ac.be
}

\section{Summary}

Amphiphilic macromolecules combining a biodegradable and a bioeliminable block have been synthesized in order to build micellar drug delivery systems. Particularly, redox reversible disulfide linkages have been introduced in the structure to ensure cross-linking of the micelles formed in water and prevent their dissociation at high dilution upon injection. The synthesis of polymers and their micellization will be especially emphasized in this paper.

Key Words: biomaterials, polymer micelles, cross-linked micelles, glutathione responsive micelles.

\section{Introduction}

Over the last decade, polymer micelles have attracted an increasing interest in pharmaceutical research because they could be used as efficient drug delivery systems [1]. Micelles of amphiphilic block copolymers are supramolecular core-shell type assemblies of tens of nanometers in diameter [2]. In principle, the micelle core is usually constructed with biodegradable hydrophobic polymers such as aliphatic polyesters, e.g. poly( $\varepsilon$-caprolactone) (PCL), which serves as a reservoir for the incorporation of various lipophilic drugs. Water soluble poly(ethylene oxide) (PEO) is most frequently used to build the micelle corona because it is very efficient in preventing protein adsorption at surfaces and in stabilizing the micelles in the blood compartment, giving rise to particles invisible to the body defence system [3].

Even if micelles get a high stability in aqueous media thanks to their low critical micellar concentration, the dissociation of micelles is not always preserved when they are injected in the blood compartment. The reversible cross-linking of the core of micelles by disulfide bridges has thus been targeted to provide the stability of micelles after the administration and release the drug by intracellular reduction of the disulfide bridges due to higher concentrations of gluthatione.

For this purpose, new functional macromolecular architectures have been synthesized and used to prepare micelles for the targeting of tumor cells. Basically, disulfide bridges have been introduced in the PCL segment by a well-known click-reaction of a bis-alkyne disulfide onto PCL bearing pendent azide groups. Three kinds of cross-linking strategies based on various block-copolymer architectures have been investigated depending on whether the core of the micelles is loosely cross-linked (a) or only the periphery (b) or the center (c) are fully cross-linked (Figure 1). The synthesis and the micellization of these macromolecular structures were studied and the efficiency of preventing dissociation around healthy cells was tested.

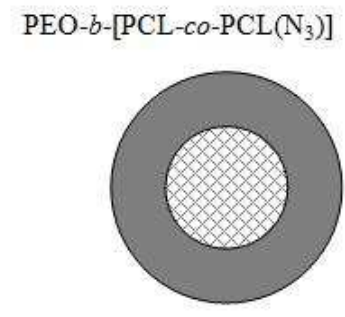

(a)

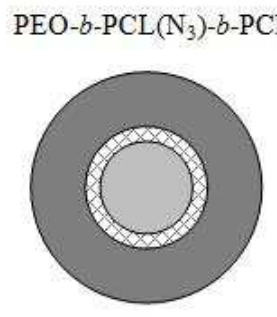

(b)

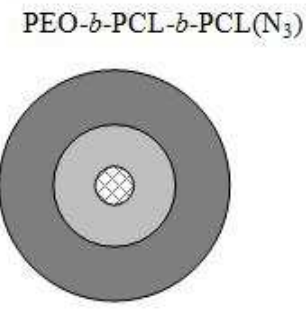

(c)

Figure 1. Structure of core cross-linked micelles versus macromolecular architecture. 


\section{Experimental Methods}

Polymer Synthesis. MPEO- $b$-[PCL-co-PCL(Cl)] (2a) was prepared by ring-opening copolymerization (ROP) of $\varepsilon$-caprolactone ( $\varepsilon-\mathrm{CL})$ and $\alpha$-chloro- $\varepsilon$-caprolactone $(\alpha-\mathrm{Cl}-\varepsilon-\mathrm{CL})$ using monomethoxypoly(ethylene glycol) (MPEO) as macroinitiator. The polymerization was performed in toluene at reflux for $48 \mathrm{~h}$ in the presence of tin octoate. The same protocol was used for the synthesis of MPEO- $b$-PCL- $b$-PCL(Cl) (3b), MPEO- $b$-PCL(Cl)- $b$-PCL (3c) by sequential polymerization. For polymer functionalization, chlorides were converted into azides by reaction of $\mathbf{2 a}, \mathbf{3 b}$ and $\mathbf{3 c}$ with sodium azide $\left(\mathrm{NaN}_{3}\right)$ in dimethylformamide (DMF) at room temperature overnight.

Formation of Micelles. A stock solution of copolymer $(\mathbf{3 a}, \mathbf{4 b}$ or $\mathbf{4 c})$ was prepared with a well-defined concentration (1\%) in DMF in presence of the cross-linking agent (Scheme 3) which was synthesized according to a previous report [4]. A solution of $20 \mathrm{~mL}$ of water was added to the organic solution (5 $\mathrm{mL}$ ) under vigorous stirring for one day. $\mathrm{CuSO}_{4}$ and sodium ascorbic acid were added to click the cross-linking agent to the copolymer. The reaction was allowed to proceed for one day at room temperature and purified by dialysis against water.

\section{Results and Discussion}

The synthesis of the three macromolecular architectures, obtained by the ring-opening polymerization of $\varepsilon-\mathrm{CL}$ and $\alpha-\mathrm{Cl}-\varepsilon-\mathrm{CL}$, is illustrated in Scheme 1. The control of the polymerization is confirmed by SEC with low polydispersity index and a shift of the elution time of the copolymer formed. Numberaverage molecular weights $\left(\mathrm{M}_{\mathrm{n}}\right)$ and composition were determined by ${ }^{1} \mathrm{H}-\mathrm{NMR}$ and are summarized in Table 1. Complete monomer conversion was obtained and the molar composition of the copolymers agreed with the composition of the comonomer feed.

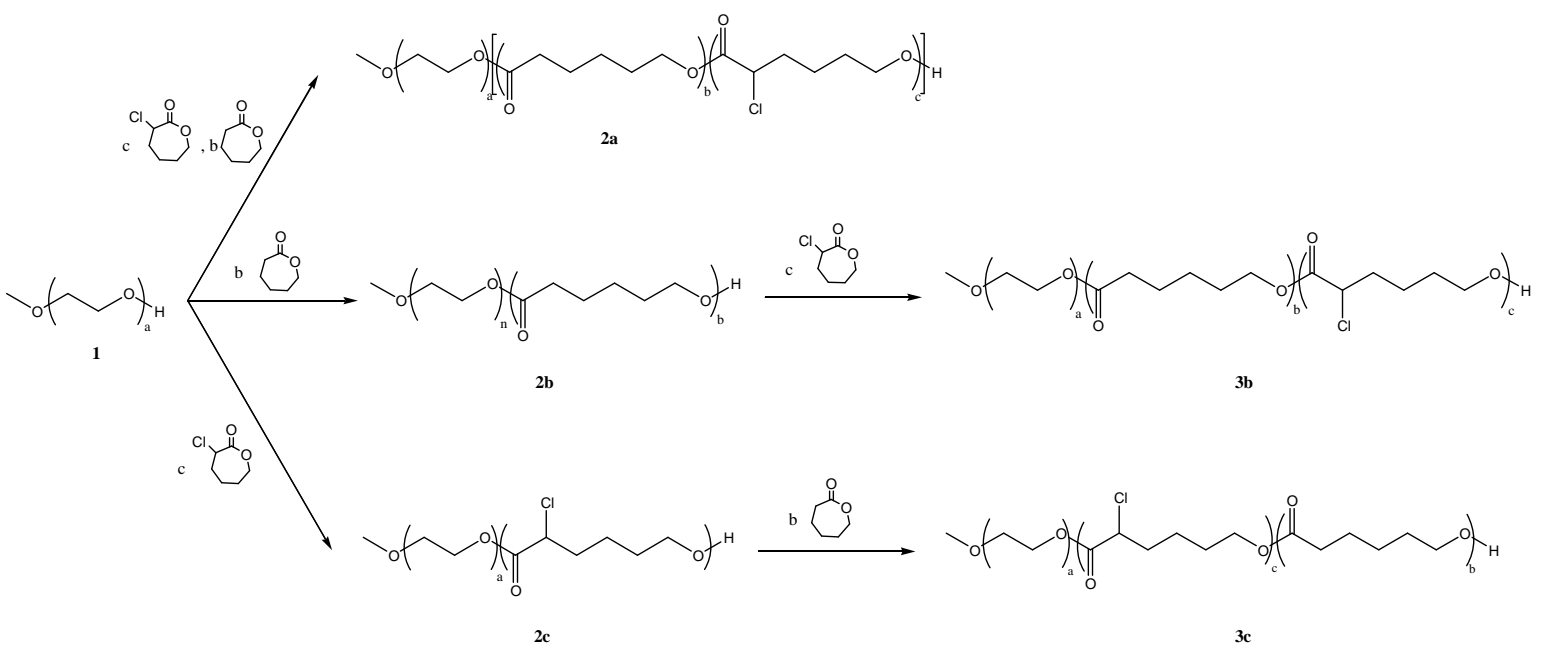

Scheme 1: Strategy for the synthesis of MPEO- $b$-[PCL-co-PCL(Cl)] (2a), MPEO- $b$-PCL- $b$-PCL(Cl) (3b), MPEO- $b$-PCL(Cl)-b-PCL (3c)

Table 1: Results obtained for the three copolymerizations of $\varepsilon-\mathrm{CL}$ and $\alpha-\mathrm{Cl}-\varepsilon-\mathrm{CL}$ from monomethoxy poly(ethylene glycol) $\mathrm{Mn}=5000 \mathrm{~g} / \mathrm{mol}$ ) by ROP.

\begin{tabular}{|c|c|c|c|c|c|c|c|}
\hline & $\begin{array}{c}M_{n, \text { th }} P C L \\
(\mathrm{~g} / \mathrm{mol})(\mathrm{DP})\end{array}$ & $\begin{array}{l}M_{n, \text { th } P(\alpha-C l-C L)} \\
(\mathrm{g} / \mathrm{mol})(\mathrm{DP})\end{array}$ & $\begin{array}{c}D P_{t h P C L} / \\
D P_{t h P(\alpha-C l-C L)}\end{array}$ & $\begin{array}{c}M_{n, N M R} P C L \\
(\mathrm{~g} / \mathrm{mol})(D P)\end{array}$ & $\begin{array}{c}M_{n, N M R P(\alpha-C l-C L)} \\
(g / m o l)(D P)\end{array}$ & $\begin{array}{c}D P_{N M R P} P(\alpha-C l-C L) \\
/ D P_{N M R P C L}\end{array}$ & $\begin{array}{r}M_{w} / M_{n} \\
(S E C)\end{array}$ \\
\hline $2 a$ & $1370(12)$ & $590(4)$ & $75 / 25$ & $1480(13)$ & $590(4)$ & $76 / 24$ & 1.08 \\
\hline $3 b$ & $1370(12)$ & $590(4)$ & $75 / 25$ & $1370(12)$ & $440(3)$ & $80 / 20$ & 1.06 \\
\hline $3 c$ & $1370(12)$ & $590(4)$ & $75 / 25$ & $1370(12)$ & $740(5)$ & $70 / 30$ & 1.05 \\
\hline
\end{tabular}

The conversion of the chloride groups to azide by reaction with sodium azide to obtain the final macromolecular architecture is illustrated in Scheme 2. The IR spectrum shows a new absorption band at $2106 \mathrm{~cm}^{-1}$ characteristic of the azide. Quantitative conversion is confirmed by ${ }^{1} \mathrm{H}-\mathrm{NMR}$ with the disappearance of the $\mathrm{C} \underline{\mathrm{HCl}}$ peak at $4.25 \mathrm{ppm}$ and the appearance of a new one at $3.85 \mathrm{ppm}\left(\mathrm{C}_{\underline{H}} \mathrm{~N}_{3}\right)$. 


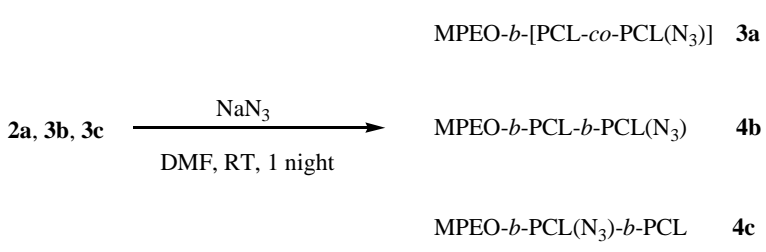

Scheme 2: Conversion of chloride to azide

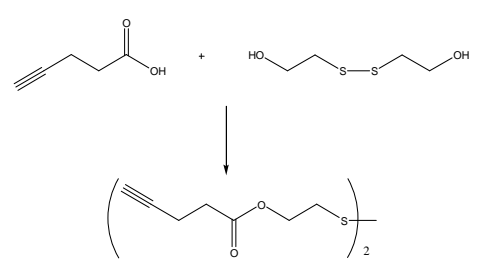

Scheme 3: Synthesis of the cross-linking agent

The micellization of these copolymers was studied by dissolving them in a good solvent of the hydrophilic and hydrophobic block and adding water. During this process, the cross-linking agent migrates to the hydrophobic core of micelles formed and, the addition of $\mathrm{CuSO}_{4}$ and sodium ascorbate salt, cross-links the core of the micelles by the Huisgen 1,3-dipolar cycloaddition of azides present on the copolymers. Micelles obtained are characterized by dynamic light scattering (DLS) and transmission electron microscopy (TEM). The hydrodynamic diameter of the micelles formed under these conditions is reported in the Table 2. The spherical shape of the micelles was confirmed by TEM (Figure 2).

To confirm the cross-linking of the micelles, they were dissolved in DMF, a good solvent of the two blocks, and analyzed by DLS. Larger particles of about $200 \mathrm{~nm}$ are observed. In the case of non-crosslinked micelles, no signal was obtained. In addition, upon reaction with an excess of free thiol, the micelles formed in DMF lost their stability confirming the reversibility of the cross-linking.

Table 1: Size distribution obtained for micelles of copolymers $3 \mathrm{a}, 4 \mathrm{~b}, 4 \mathrm{c}$.

\begin{tabular}{l|cc}
\hline \multicolumn{1}{c|}{ Sample } & Dh $(\boldsymbol{n m})$ & PDI \\
\hline MPEO- $b$-[PCL-co-PCL( $\left.\left.\mathrm{N}_{3}\right)\right](\mathbf{3 a})$ & 49.9 & 0.17 \\
MPEO- $b$-PCL- $b$-PCL $\left(\mathrm{N}_{3}\right)$ (4b) & 40.6 & 0.14 \\
MPEO- $b$-PCL( $\left.\mathrm{N}_{3}\right)-b$-PCL (4c) & 53.8 & 0.2 \\
\hline
\end{tabular}

\section{Conclusion}

Three new macromolecular architectures have been prepared for drug delivery systems. Micelles of these copolymers are nano-objects of about $40-50 \mathrm{~nm}$. The cross-linking by disulfide bridges provided them the stability to be injected in the blood. In the future, cytotoxicity and cellular uptake of the micelles will be investigated further so as drug incorporation and release.

\section{Acknowledgements}

S.C. is grateful to the Belgian "Fond pour la Formation à la Recherche dans l'Industrie et dans l'Agriculture » (FRIA) for financial Support. The authors are much indebted to IAP VI-27 «Functional Supramolecular systems" (FS2).

\section{References}

[1] K. Kataoka, A. Harada, Y. Nagasaki, Block copolymer micelles for drug delivery: design, characterization and biological significance, Adv. Drug Deliv. Rev. 47(1) (2001) 113-131.

[3] K. Van Butsele, R. Jérôme, C. Jérôme, Functional amphiphilic and biodegradable copolymers for intravenous vectorization, Polymer 48(26) (2007) 7431-7443.

[4] C. Jérôme, P. Lecomte, Recent advances in the synthesis of aliphatic polyesters by ring-opening polymerization, Adv. Drug Deliv. Rev. 60(9) (2008) 1056-107.

[5] H. Gao, K.Matyjaszewski, Synthesis of star polymer by a combination of ATRP and the "click" coupling method, Macromolecules 39 (2006) 4960-4965. 